\title{
New developments in goblet cell mucus secretion and function
}

\author{
GMH Birchenough $^{1}$, MEV Johansson ${ }^{1}$, JK Gustafsson ${ }^{1}, \mathrm{JH}_{\text {Bergström }}{ }^{1}$ and GC Hansson ${ }^{1}$
}

Goblet cells and their main secretory product, mucus, have long been poorly appreciated; however, recent discoveries have changed this and placed these cells at the center stage of our understanding of mucosal biology and the immunology of the intestinal tract. The mucus system differs substantially between the small and large intestine, although it is built around MUC2 mucin polymers in both cases. Furthermore, that goblet cells and the regulation of their secretion also differ between these two parts of the intestine is of fundamental importance for a better understanding of mucosal immunology. There are several types of goblet cell that can be delineated based on their location and function. The surface colonic goblet cells secrete continuously to maintain the inner mucus layer, whereas goblet cells of the colonic and small intestinal crypts secrete upon stimulation, for example, after endocytosis or in response to acetyl choline. However, despite much progress in recent years, our understanding of goblet cell function and regulation is still in its infancy.

\section{THE INTESTINE}

The gastrointestinal tract is a remarkable organ. Not only can it digest most of our food into small components, but it is also filled with kilograms of microbes that live in stable equilibrium with us and our immune system. A digestive system can be traced back to early metazoans and Cambrian evolution, ${ }^{1}$ long before an adaptive immune system developed. The first appearance of molecules with protein domains typical to polymeric mucins was among metazoans as was the appearance of glycosyltransferases necessary for decorating the mucin protein core. ${ }^{2,3}$ Together, this allowed the biosynthesis of molecules that are virtually indigestible by the host's digestive enzymes. Avoiding self-digestion is a primary prerequisite of a digestive system, but handling of the luminal content without triggering an overt immune reaction while generating tolerance requires an intricate balance that is still poorly understood. A better understanding of epithelial cells and their function as part of the innate immune system, as well as their communication with lamina propria and intraepithelial adaptive immune cells, are a likely source of further understanding. ${ }^{4}$

The single layer of epithelial cells along the gastrointestinal tract is very active and takes part in different absorption and secretion processes. To be able to withstand the physical and mechanical stress placed on these cells, there is a continuous system of mucus covering the epithelium. There is a two-layered mucus system in the stomach and colon and a single-layered mucus in the small intestine. ${ }^{5}$ The mucus layers in these three regions perform their protective function using different mechanisms and must be considered separately in order to properly comprehend the overall mucus system, a consideration that is often overlooked in the immunological literature.

\section{THE PRINCIPLE OF SEPARATION}

For quite some time the importance of the mucus covering the epithelium was overlooked and mucosal immunologists instead attempted to understand how the immune system could tolerate luminal bacteria that were assumed to be in direct contact with epithelial cells. However, this assumption was changed by the observation of an inner colonic mucus layer separating the bacteria from the host epithelium. ${ }^{6-8}$ Further studies of the gastrointestinal mucus systems have shown that all mucus functions to separate the luminal content, especially bacteria, from direct contact with the epithelial cells. The epithelial cells and especially the enterocytes are providing the best separation of luminal material from the lamina propria. Of special importance is the enterocyte apical glycocalyx that is built by transmembrane mucins and the tight junctions that

${ }^{1}$ Department of Medical Biochemistry, University of Gothenburg, Gothenburg, Sweden. Correspondence: GC Hansson (gunnar.hansson@medkem.gu.se) Received 16 December 2014; accepted 20 March 2015; published online 15 April 2015. doi:10.1038/mi.2015.32 
firmly anchor the cells to each other. ${ }^{9,10}$ These systems are of course not perfect and some bacteria can come in contact with the epithelium even in the healthy intestine. However, the mucosal immune system is programmed to tolerate low numbers of bacteria as exemplified by macrophage populations in the colon that can engulf bacteria, but still do not induce inflammatory responses or activate the adaptive immune system. ${ }^{11,12}$

The net-like mucins forming the intestinal mucus have different properties in the small and large intestine. In the small intestine the pore sizes are large, allowing bacteria and bacteriasized particles to penetrate the mucus. ${ }^{5}$ The mucus fills the space between the villi and covers the villi tips, but bacteria are typically not found in contact with the epithelium except at the villus tip. The carbohydrate-rich polymeric mucin binds water that limits and slows down diffusion. The antibacterial peptides and proteins secreted from crypt Paneth cells and enterocytes into the mucus are of major importance for keeping bacteria at a distance. ${ }^{13-15}$ However, without the mucus, these antibacterial components would quickly be diluted into the intestinal lumen. The mucus concentrates them near the epithelium and thus generates an antibacterial gradient from the epithelial cells out toward the lumen. The mucus also slows down bacterial penetration and is continuously renewed by the goblet cells, a process that pushes bacteria out toward the lumen. Together, this explains the lack of bacteria in contact with the epithelial cells in the small intestine and may also suggest why most pathogenic bacteria are motile, i.e., in order to swim against the mucus flow and overcome the diffusion barrier. This penetrability of the small intestinal mucus may be the reason why pathogenic bacteria mostly infect this region of the gut using different mechanisms as discussed elsewhere. ${ }^{16}$

The small intestinal mucus is normally easily aspirated and nonattached. However, this is not the case in germ-free animals where the MUC2 mucin, and thus the mucus, remains anchored to the goblet cells. The reason for this is that a proteolytic enzyme, meprin $\beta$, is required for cleaving MUC2 and releasing it from the goblet cell attachment. ${ }^{17}$ Meprin $\beta$ is anchored in the enterocyte apical membrane and cannot access the mucin goblet cell anchor from this position. To contact MUC2 it needs to first be released by extracellular cleavage, allowing it to diffuse into the mucus. The release of anchored meprin $\beta$ is controlled and triggered by exposure to bacteria. This regulation of the mechanism for releasing anchored mucus suggests that it has an important role in the small intestine's handling of bacteria. A hint of this function can be observed in the small intestine lacking a functional CFTR chloride and bicarbonate channel as in the disease cystic fibrosis. ${ }^{18}$ In this case the mucins also remain anchored as low levels of bicarbonate do not sufficiently well remove calcium ions to allow for unfolding the packed MUC2, necessary for exposing the meprin $\beta$ cleavage sites. ${ }^{17}$ In mice and humans a nonfunctional CFTR channel causes bacterial overgrowth, distal intestinal obstruction syndrome, and sometimes obstructive ileus. This shows how important mucus is for trapping and moving bacteria away from the host surface.
In the colon there is a two-layered mucus system where the inner mucus layer is stratified and organized as a filter that physically separates the bacteria from the epithelial cells. ${ }^{6}$ This layer is anchored to the goblet cells. At $\sim 50 \mu \mathrm{m}$ (mouse) or $200 \mu \mathrm{m}$ (human) distance from the epithelium, endogenous protease activities convert the inner mucus layer into the outer nonattached mucus that is slowly expanded $2-3$-fold in volume. This expansion increases the pore sizes, allowing bacteria to penetrate. The pore sizes of the inner mucus layer are sufficiently small to hinder penetration of bacteria or beads down to $0.5 \mu \mathrm{m}$ diameter. Interestingly, this penetrability is regulated. Germ-free animals have a penetrable mucus as is also the case for animals lacking the cytokine interleukin (IL)-10 or the NHE3 (sodium-hydrogen exchanger 3) ion transporter. ${ }^{19}$ The composition of the bacterial flora can also influence the mucus penetrability as observed in genetically identical animals. ${ }^{20}$ The molecular mechanisms underlying this modulation of inner mucus penetrability are not yet understood.

In the absence of MUC2 there is no mucus and bacteria are in direct contact with the epithelium, something that triggers inflammation similar to that found in ulcerative colitis and, in the long term, can lead to cancer. Furthermore, animals with a penetrable inner mucus layer develop spontaneous colitis. Patients with active ulcerative colitis have a fully penetrable mucus, whereas patients in remission have a more variable profile with some looking perfectly normal. ${ }^{19}$ Together, this demonstrates the importance of separating the majority of the luminal contents from the epithelial cells, a function that is dependent on the mucus layer.

\section{THE MUCINS ARE THE STRUCTURAL BUILDING BLOCK IN MUCUS}

The intestinal mucus proteome is $\sim 50$ proteins with a few that are very abundant. The major component is the MUC2 mucin, the function of which has been determined in recent years. However, the functions of most of the other more abundant proteins, such as FCGBP, CLCA1, AGR2, and ZG16, remain mostly unknown. ${ }^{6}$ MUC2 comprises $\sim 5,100$ amino acids, with the uncertainty originating from the central region that is still not fully sequenced. ${ }^{21}$ MUC2 contains two long central regions called PTS domains that become rigid, outstretched rods after $\mathrm{O}$-glycosylation and are referred to as mucin domains (Figure 1). In MUC2 these regions are $\sim 0.5 \mu \mathrm{m}$ long. The $\mathrm{N}$ - and C-terminal regions are $\sim 1,300$ and 1,000 amino acids, respectively, and are tightly folded and stabilized by numerous disulfide bonds. The MUC2 monomeric building block has a mass of $\sim 2.5 \mathrm{MDa}$ when fully glycosylated and is polymerized by C-terminal dimerization and $\mathrm{N}$-terminal trimerization, resulting in the formation of enormous net-like polymeric sheets. $^{22}$

\section{GOBLET CELLS}

Goblet cells are specialized for the synthesis and secretion of mucus. They acquired their name for their typical goblet, cuplike, appearance formed by the mucin granulae that fill up the cytoplasm (Figure 1). In addition to MUC2, the mucin 


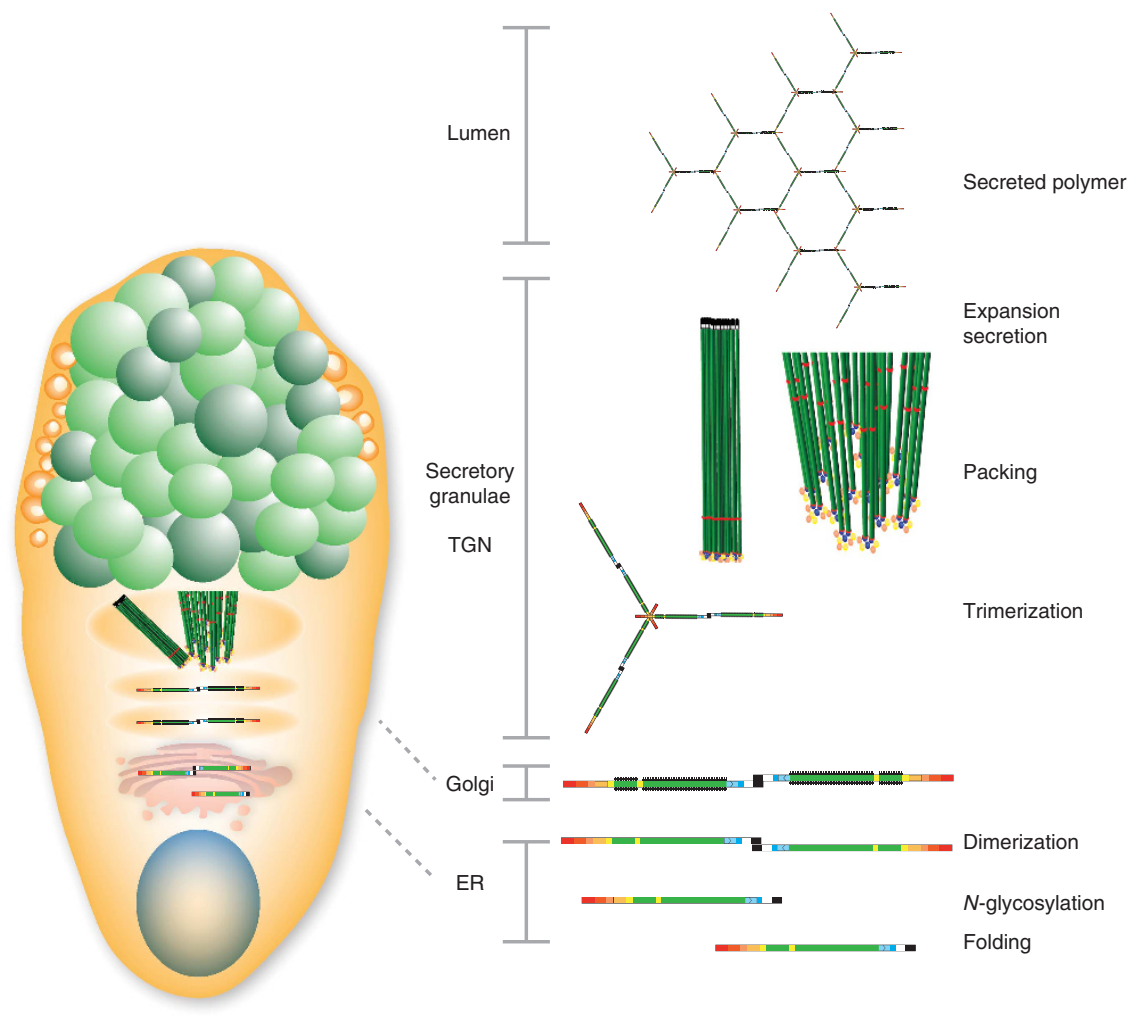

Figure 1 Assembly of the MUC2 mucin in the goblet cell. ER, endoplasmic reticulum; TGN, trans-Golgi network.

granulae are filled with the other typical mucus components FCGBP, CLCA1, ZG16, and AGR2. Mice lacking Muc2 have the same number of goblet cells but these do not have the typical goblet cell shape.

The intestinal surface epithelium, including goblet cells, is continuously renewed from the stem cells at the crypt base with normal cell turnover between 3 and 7 days. ${ }^{23}$ The stem cells give rise to all epithelial cells including the enterocytes, goblet cells, Paneth cells, enteroendocrine cells, and Tuft cells. The enterocyte lineage is the primary cell lineage and its differentiation pathway is controlled by Notch signaling. When Notch signaling is blocked by $\gamma$-secretase inhibition, the Notch cytoplasmic component no longer enters the nucleus, inhibiting transcriptional signaling and shifting differentiation into the secretory pathway. ${ }^{24}$ Without further changes in signaling, goblet cells are the default result of this shift. Furthermore, the transcription factor SPDEF (SAM pointed domain ETS factor) is important for full maturation into goblet cells. ${ }^{25}$

Although there is still limited knowledge of the cell biology of goblet cells, it has become obvious that there are several types that function in different ways. In the small intestine the goblet cell adjacent enterocytes supply the required bicarbonate for proper mucin unfolding. ${ }^{18}$ This is not the case in the large intestine where the goblet cell might supply bicarbonate via its own bestrophin-2 bicarbonate transporter. ${ }^{26}$ Other examples are the intercrypt colonic surface goblet cells that continuously secrete mucus and by this build the inner mucus layer contrasts with the goblet cells in the upper part of the colonic crypts that can secrete by rapid compound exocytosis. ${ }^{27,28}$ In order to better understand goblet cell secretion and function we first need to realize that these cells are more diverse and capable than has been previously anticipated.

\section{GOBLET CELL EXOCYTOSIS}

MUC2 polymers are densely packed in the regulated secretory vesicles of the goblet cell because of the low $\mathrm{pH}$ and high calcium in these compartments. In this milieu the N-terminal trimers spontaneously form concatenated rings with the long extended mucin domains standing perpendicular to this structure and joined to other MUC2 molecules end to end by their C-termini (Figure 1). ${ }^{22}$ More recent structural studies of the MUC2 $\mathrm{N}$-terminal D3 domain suggested that every second MUC2 mucin layer is rotated $180^{\circ}$ and the $\mathrm{N}$-terminal ring structures meet. ${ }^{29}$ Upon secretion, the densely packed mucin expands $>1,000$-fold into large nets (Figure 1). This process requires an increase in $\mathrm{pH}$ to and the removal of $\mathrm{N}$-terminally bound single calcium ion. ${ }^{30}$ Bicarbonate is the ideal and physiological solution for precipitating calcium and raising the $\mathrm{pH}^{18}$ Previous models of mucin packing in the goblet cell have suggested that this is less organized and that the calcium only acts as a shield preventing electrostatic repulsion by negatively charged glycans; however, this model does not allow for the rapid and well-organized mucin expansion observed. $^{31}$ 
Secretion of mucins can take place in at least two ways: regulated vesicle secretion and compound exocytosis. Regulated vesicle secretion has been well studied in airway cells. ${ }^{32}$ This process includes the fusion of single vesicles with the plasma membrane mediated by typical vesicle exocytosis components like Syntaxins, Munc18, VAMP, and SNAP proteins. Secretion from surface goblet cells of both the small and large intestine, although not studied, probably follows similar mechanisms. Compound exocytosis is a more dramatic event where most of the vesicles that make up the goblet cell theca fuse and empty their content. There are no molecular studies on this type of secretion, but there are very clear morphological studies that show that the whole cell essentially explodes during this secretory process. ${ }^{33}$

Renewal of the colonic inner mucus layer by mucus secretion is important for its protective function. Mucus renewal is much faster than epithelial cell turnover and is most likely tightly coupled to propulsion of the luminal material. Turnover studies have been conducted by in vivo glycoprotein labeling using an azide-modified $\mathrm{N}$-acetylgalactosamine, GalNAz, that is incorporated into glycans during protein biosynthesis. ${ }^{27}$ Mucins, with their numerous $O$-glycans, are very effectively labeled by this method. The goblet cells on the colon surface epithelium showed rapid mucin synthesis and secrete the mucus without extensive storage. This was in contrast to the goblet cells in the upper crypts, where the mucus accumulated in granulae over time and secretion was slower. In the small intestine, with its larger epithelial surface, cell renewal takes between 3 and 5 days. ${ }^{23}$ Investigation of this region using the same method demonstrated that crypt goblet cells were labeled very slowly, but massive secretion was observed at the crypt opening a few hours after GalNAz injection, suggesting an enforced protection of the vulnerable crypts. ${ }^{27}$ Conversely, the goblet cells on the villi have faster mucin biosynthesis and secretion.

\section{GOBLET CELL ENDOCYTOSIS}

Recent research has indicated that optimal intestinal goblet cell mucus exocytosis is dependent on several intersecting cellular processes that together appear to modulate mucin granule accumulation and secretion. These include endocytosis, autophagy, reactive oxygen species (ROS) generation, and inflammasome assembly and activation.

Endocytosis of luminal material by colonic goblet cells was described in the 1980s by showing the uptake of cationic ferritin using electron microscopy. ${ }^{34,35}$ These studies observed that in some goblet cells intracellular cationic ferritin was localized to endosomes located at the periphery of the theca and that these vesicles eventually fused with lysosomes. Although the extent of endocytosis was relatively limited in adult tissues, it was substantially elevated in fetal tissue indicating a developmental aspect to this process. ${ }^{35}$ At the time it was believed that this was a mechanism for recycling the plasma membrane that accumulated apically during exocytosis; ${ }^{36}$ however, the endocytotic pathway has recently been shown to have a function beyond membrane salvage.

Using a novel method for culturing differentiated intestinal cells from mouse stem cells, Patel et al. ${ }^{37}$ demonstrated that inhibition of clathrin-mediated endocytosis resulted in the accumulation of goblet cell mucin granulae. Importantly, a similar phenotype was observed in cells lacking proteins involved in conventional autophagy including Atg5, Atg14, and FIP200. Mucin accumulation occurred independently of mucin expression, indicating that the effect was likely secondary to defects in mucin secretion. Electron microscopy showed that LC3 $\beta$, a marker for autophagosomes, could be localized to multivesicular vacuoles that were also positive for the endosomal marker EEA1. This association was abrogated in endocytosis-inhibited cells, thus potentially linking the autophagy and endocytotic machinery to mucin secretion in goblet cells.

Autophagy is a ubiquitous cytoplasmic process in which a double-membrane autophagosome is formed and subsequently fused with lysosomes for degradation of enclosed components, a process linked to immunity and inflammation. ${ }^{38}$ An autophagosome can also fuse with endosomes to form intermediate structures called amphisomes that closely resemble the LC3 $\beta /$ EEA1-positive vacuoles observed by Patel et al. ${ }^{37}$ This study also provided evidence that these amphisome-like structures possess nicotinamide adenine dinucleotide phosphate oxidases and that their loss, due to inhibition of endocytosis or knockout of autophagy genes, results in decreased cellular ROS production. Crucially, application of exogenous ROS rescued the mucin accumulation phenotype, indicating that ROS may be involved in regulating mucin secretion.

Another potential actor in the regulation of goblet cell secretion is the inflammasome. These are multiprotein complexes that typically comprise one of the NOD-like receptor (NLR) family members linked to an inflammatory caspase, such as Caspase 1, via interactions with the adapter protein ASC (apoptosis-associated speck-like protein containing a CARD). ${ }^{39}$ NLRPs are thought to function as sensors for various motifs in pathogen-associated molecules and for endogenous damage or danger signals. Upon activation, NLRPs bind ASC that in turn recruits Procaspase 1. NLRPs oligomerize via their NACHT domains, resulting in a large ring-shaped inflammasome complex with seven- or eight-fold symmetry. Inflammasome assembly permits proximityinduced autoactivation of inflammatory caspases that classically proceed to cleave the proforms of IL- $1 \alpha$, IL- $1 \beta$, and IL- 18 cytokines into their mature active forms. ${ }^{40}$ Inflammasomes are abundantly expressed among immune cells and most current knowledge is derived from studies in these cell types; however, some NLRPs are also found in mucosal epithelial cells and are suggested to have roles in intestinal homeostasis. ${ }^{41-44}$

NLRP6 is expressed by colonic goblet cells and was recently suggested to have a functional role in mucin exocytosis as mice lacking NLRP6 displayed a similar mucin granule accumulation as reported for Atg5 ${ }^{-1-}$ intestinal cells. ${ }^{37,44}$ Lack of NLRP6 was previously shown to have functional consequences in vivo as knockout mice were more susceptible to both dextran sodium sulfate- and Citrobacter rodentium-induced colitis. ${ }^{41,44}$ Similar outcomes were observed in both Caspase $1 / 11^{-1-}$ and 
$\mathrm{ASC}^{-1-}$ animals, implying that activated and fully assembled inflammasomes are required for functional mucus secretion. In addition, mice lacking NLRP6 had increased numbers of damaged mitochondria and alterations in autophagic flux, both of which are symptomatic of defective autophagy.

Current data suggest that there are strong links between endocytosis, autophagy, inflammasome activity, ROS generation, and mucin secretion. Understanding how these systems are coupled together and the molecular basis for their regulatory effect on goblet cell exocytosis will be interesting avenues of future research. However, it is already clear that mucus secretion is an end point of the interaction of all of these innate immune system molecules, once more pointing to the central role of mucus secretion and the generation of a functional mucus layer in protecting the host epithelium.

\section{INDUCED GOBLET CELL MUCUS SECRETION}

Exocytosis of mucin granulae can also be regulated by intracellular $\mathrm{Ca}^{2+}$ levels and $\mathrm{Ca}^{2+}$ mobilizing agents such as acetylcholine and histamine are potent inducers of intestinal mucus secretion. Acetylcholine is by far the most studied mucus secretagogue and has been shown to induce mucus secretion in both the small and large intestine of mouse, rat, and rabbit, and in the human colon. ${ }^{5,33,45-47}$ Stimulation with acetylcholine or other cholinergic agonists such as carbachol results in a rapid transient increase in mucus secretion rates that reverts to baseline values within $30 \mathrm{~min} .{ }^{47}$ Acetylcholine primarily targets the goblet cells in the small intestinal crypts, resulting in more or less complete emptying of the stored mucin granule pool. The villus goblet cells in the small intestine and the surface goblet cells in the colon do not respond to acetylcholine or carbachol with exocytosis. Although cholinergic agonists are inducers of mucus secretion in both the small and large intestine, the small intestine is by far most sensitive to carbachol. ${ }^{5,47}$ Conversely, the opposite pattern is instead true for histamine that induces mucus secretion in the colon, but not in the small intestine. ${ }^{45,48}$ The immune modulator prostaglandin $E_{2}$ has been shown to induce mucus secretion in rat colon and mouse small intestine, but only induces fluid secretion in the human colon. ${ }^{48,49}$ Species and segmental differences in the responsiveness to prostaglandin $\mathrm{E}_{2}$ may be explained by it being able to signal via both cyclic adenosine monophosphate- and $\mathrm{Ca}^{2+}$-dependent pathways depending on which of its receptors is present.

The triggers for compound exocytosis from the colonic crypts have not yet been clearly identified. However, a coordinated process of mucus secretion, ion and fluid secretion, and motility is an efficient way to dispose harmful agents. The importance of this secretory process in maintaining the intestinal barrier was recently described by Grootjans et al..$^{50}$ who showed that ischemia-induced tissue damage was prevented by compound exocytosis of mucin vesicles in the colonic crypt goblet cells that cleared the crypts of bacteria that had managed to penetrate the crypts during the ischemic period.

\section{GOBLET CELLS AS ANTIGEN IMPORTERS}

Although production and secretion of mucus are the main functions of the intestinal goblet cells, recent studies have shown that the small intestinal population is also able to acquire luminal antigens and present these to $\mathrm{CD}_{103}{ }^{+}$dendritic cells in the lamina propria. ${ }^{51,52}$ Spontaneous antigen uptake was observed in the small intestine of conventionally raised mice, but not in the colon. However, in mice raised under germ-free conditions and in conventionally raised mice following oral treatment with antibiotics, spontaneous antigen uptake was also observed in the colon, suggesting that the uptake process is inhibited by the colonic microbiota. ${ }^{51,53}$ Similar to regulation of mucus secretion, antigen uptake by the small intestinal goblet cells is augmented by cholinergic agonists and both baseline and agonist-induced antigen uptake is mediated via muscarinic receptor $4 .{ }^{53}$ The ability of the intestinal goblet cells to acquire luminal antigens correlates with the expression of the $\mathrm{m} 4$ receptor, i.e., higher expression in the small intestine compared with the colon and increased expression following antibiotics treatment. ${ }^{53}$ The observed differences in carbachol-induced antigen uptake in the small and large intestine correlate with the observed differences in the sensitivity to carbachol-induced mucus secretion, suggesting that the two processes are regulated by similar mechanisms.

\section{IMMUNE REGULATION OF GOBLET CELL FUNCTION}

That the intestinal goblet cells are under direct regulation by the immune system is best exemplified by the striking goblet cell hyperplasia and mucus hypersecretion associated with parasitic helminth infections. ${ }^{54,55}$ These infections elicit a Thelper type 2 (Th2) response with increased levels of cytokines such as interleukin IL-4, IL-5, IL-9, and IL-13, where IL-13 is considered the major effector cytokine. Intestinal epithelial cells have been shown to express the IL- $4 \mathrm{R} \alpha$ and IL13R $\alpha 1$ subunits, making it likely that IL-13 acts directly on the epithelium to induce goblet cell hyperplasia via STAT6 (signal transducer and activator of transcription-6) signaling. ${ }^{56}$ Further support in favor of IL-13 being an important regulator of goblet cell hyperplasia is found in studies showing that IL-13overexpressing mice develop intestinal goblet cell hyperplasia and that exogenous IL-25 or IL-9 overexpression induced goblet cell hyperplasia and increased mucin expression via IL-13-dependent pathways. ${ }^{57} \mathrm{Th} 2$ responses, including goblet cell hyperplasia, are also fundamental to allergic asthma. Interestingly, recent observations suggest that the SPDEF transcription factor is required for the normal pulmonary Th2 inflammatory response and goblet cell hyperplasia. ${ }^{58}$ SPDEF regulates goblet cell maturation and differentiation in both the lung and intestine, ${ }^{25,58,59}$ further suggesting that the goblet cell is involved in the regulation of the immune system. ${ }^{60}$

In addition to the importance of Th2 cytokines in regulation of goblet cell function, recent findings also implicate the Th17associated cytokine IL-22 in regulation of goblet cell differentiation and mucin expression as IL-22-deficient mice fail to increase Muc2 expression and have reduced levels of goblet cell hyperplasia in response to Nippostrongylus brasiliensis and 
Trichuris muris infections compared with wild-type animals. ${ }^{61}$ Interestingly, these effects were observed in the presence of increased levels of IL-4 and IL-13, suggesting overlapping pathways for induction of mucin expression and goblet cell differentiation. Less is understood on the role of Th1 cytokines such as interferon- $\gamma$ and tumor necrosis factor- $\alpha$, and Th17 cytokines such as IL-23 and IL-17 in regulation of goblet cell function.

\section{REGULATION OF GOBLET CELLS BY IMMUNOMODULATORY CYTOKINES}

The MUC2 mucin is not only large, but also has termini that are stabilized by disulfide bonding between the cysteine residues that comprise $>10 \%$ of the amino acids present in these regions. Correct folding of the terminal regions requires the careful joining of each disulfide bond, making it one of the more difficult proteins for the endoplasmic reticulum to handle. This is reflected in the need for MUC2 to have a special chaperone, ERN2 (also called IRE1 $\beta$ ), that seems to be utilized exclusively for mucin folding. ${ }^{62,63}$ Misfolded proteins trigger an unfolded protein response (UPR) that is in turn coupled to inflammation. ${ }^{64}$ Single mutations in mouse Muc2 can cause endoplasmic reticulum accumulation that triggers a UPR response and increased levels of inflammatory cytokines. ${ }^{65,66}$ Higher quantitative demands for MUC2 synthesis, such as an increased bacterial load in contact with the epithelium, will further challenge the endoplasmic reticulum folding system and trigger UPR responses and inflammation. However, there seem to be at least two immunological systems that function to limit such deleterious developments. These are based on the protective cytokines IL-22 and IL-10.

IL-22, which is primarily produced by natural killer cells, has protective effects on intestinal epithelial cells. ${ }^{67,68}$ The mechanism for this is not yet known, but recent observations on the effects of IL-22 on insulin-producing cells suggests that IL22 can reduce and alleviate inflammation in conjunction with the UPR. ${ }^{69}$

IL-10 has an immune regulatory function and is critical for intestinal homeostasis as IL-10 and IL-10 receptor-deficient mice develop colitis that is dependent on bacteria. ${ }^{70-72}$ Genome-wide association studies in inflammatory bowel disease patients have associated single-nucleotide polymorphisms in the IL10 loci with inflammatory bowel disease, ${ }^{73,74}$ and mutations in the IL-10 receptors are also linked to inflammatory bowel disease. ${ }^{75} \mathrm{IL}-10$ is produced by several cells of the immune system but has also been shown to be produced by the epithelium. ${ }^{76,77}$ The effect of this cytokine has mainly been studied in the econtext of its effect on immune cells, and its effects on epithelial cells are less explored. Interestingly, bacteria manipulated to express IL-10 have been shown to reduce experimental colitis. ${ }^{78} \mathrm{IL}-10$ has a direct effect on goblet cells by modulating their ability to manage mucin biosynthesis and misfolding ${ }^{79}$ and decreased MUC2 production has been demonstrated in IL-10-deficient mice. ${ }^{80}$ In young IL-10deficient mice, before the development of inflammation, the inner colonic mucus layer has a normal thickness, but is fully penetrable to bacteria and beads the size of bacteria. ${ }^{19}$ The bacteria in contact with the epithelium likely trigger inflammatory responses, leading to colitis as seen in mice lacking the Muc2 mucin. ${ }^{6}$ Together, it can be suggested that IL-10 and IL-22 have important positive effects on the goblet cell function, again suggesting important connections between the immune system and goblet cells.

\section{CONCLUSIONS}

Goblet cells form the major line of defense at the intestinal mucosa, based primarily on secretion of the MUC2 mucin. Although all goblet cells have a common secretory role, evidence suggests that cells in different segments of the intestine, and different segmental subregions, are responsive to variable stimuli and have distinct roles to play in maintaining intestinal homeostasis. Although the existence of different goblet cell populations is only now becoming clear, it should not be considered surprising given the diversity of other cell types of the innate immune system. Indeed, the findings summarized in this review demonstrate that immune system-associated intracellular processes and intercellular signaling have a significant role to play in regulating goblet cell activity, strongly indicating that these cells have a central role to play as the gatekeepers of the mucosal immune system.

\section{ACKNOWLEDGMENTS}

Without the hard work of all the members of the Mucin Biology Groups, this contribution would not be possible. This work was supported by the Swedish Research Council, The Swedish Cancer Foundation, The Knut and Alice Wallenberg Foundation, IngaBritt and Arne Lundberg Foundation, Sahlgren's University Hospital (LUA-ALF), Wilhelm and Martina Lundgren's Foundation, Torsten och Ragnar Söderbergs Stiftelser, The Sahlgrenska Academy, National Institute of Allergy and Infectious Diseases (U01Al095473, the content is solely the responsibility of the authors and does not necessarily represent the official views of the NIH), The Swedish Foundation for Strategic Research - The Mucus-Bacteria-Colitis Center (MBC) of the Innate Immunity Program, The Swedish CF Foundation, Erica Lederhausen's Foundation, and Lederhausen's Center for CF Research at University of Gothenburg. We also acknowledge the Mammalian Protein Expression Core Facility and the Centre for Cellular Imaging at the University of Gothenburg.

\section{DISCLOSURE}

The authors declared no conflict of interest.

c 2015 Society for Mucosal Immunology

\section{REFERENCES}

1. Erwin, D.H. \& Valentine, J.W. The Cambrian Explosion: The Construction of Animal Diversity. Roberts and Companry Publishers, Greenwood Village, CO, (2013).

2. Lang, T., Hansson, G.C. \& Samuelsson, T. Gel-forming mucins appeared early in metazoan evolution. Proc. Natl. Acad. Sci. USA 104, 16209-16214 (2007).

3. Bennett, E.P., Mandel, U., Clausen, H., Gerken, T.A., Fritz, T.A. \& Tabak, L.A. Control of mucin-type O-glycosylation: a classification of the polypeptide GalNAc-transferase gene family. Glycobiology 22, 736-756 (2012).

4. Swamy, M., Jamora, C., Havran, W. \& Hayday, A. Epithelial decision makers: in search of the 'epimmunome'. Nat. Immunol. 11, 656-665 (2010).

5. Ermund, A., Schutte, A., Johansson, M.E.V., Gustafsson, J.K. \& Hansson, G.C. Studies of mucus in mouse stomach, small intestine, and colon. I. Gastrointestinal mucus layers have different properties depending on 
location as well as over the Peyer's patches. Am. J. Physiol. Gastroint. Liver Physiol 305, G341-G347 (2013).

6. Johansson, M.E.V., Phillipson, M., Petersson, J., Holm, L., Velcich, A. \& Hansson, G.C. The inner of the two Muc2 mucin dependent mucus layers in colon is devoid of bacteria. Proc. Natl. Acad. Sci. USA 105, 15064-15069 (2008).

7. Swidsinski, A. et al. Viscosity gradient within the mucus layer determines the mucosal barrier function and the spatial organization of the intestinal microbiota. Inflam. Bowel Dis. 13, 693-670 (2007).

8. van der Waaij, L.A. et al. Bacterial population analysis of human colon and terminal ileum biopsies with $16 \mathrm{~S}$ rRNA-based fluorescent probes: commensal bacteria live in suspension and have no direct contact with epithelial cells. Inflamm. Bowel Dis. 11, 865-871 (2005).

9. Sheng, Y.H. et al. MUC1 and MUC13 differentially regulate epithelial inflammation in response to inflammatory and infectious stimuli. Mucosal Immunol. 6, 557-568 (2013).

10. Turner, J.R. Intestinal mucosal barrier function in health and disease. Nat. Rev. Immunol. 9, 799-809 (2009).

11. Bain, C.C. et al. Resident and pro-inflammatory macrophages in the colon represent alternative context-dependent fates of the same Ly6Chi monocyte precursors. Mucosal Immunol. 6, 498-510 (2013).

12. Grainger, J.R. et al. Inflammatory monocytes regulate pathologic responses to commensals during acute gastrointestinal infection. Nat. Med. 19, 713-721 (2013).

13. Vaishnava, S. et al. The antibacterial lectin Reglllg promotes the spatial segregation of microbiota and host in the intestine. Science 33, 255-258 (2011).

14. Johansson, M.E.V. \& Hansson, G.C. Keeping bacteria at a distance. Science 334, 182-183 (2011).

15. Clevers, H.C. \& Bevins, C.L. Paneth cells: maestros of the small intestinal crypts. Annu. Rev. Physiol. 75, 289-311 (2013).

16. McGuckin, M.A., Linden, S.K., Sutton, P. \& Florin, T.H. Mucin dynamics and enteric pathogens. Nat. Rev. Micro. 9, 265-278 (2011).

17. Schütte, A. et al. Microbial induced meprin $\beta$ cleavage in MUC2 mucin and functional CFTR Channel are required to release anchored small intestinal mucus. Proc. Natl. Acad. Sci. USA 111, 12396-12401 (2014).

18. Gustafsson, J.K. et al. Bicarbonate and functional CFTR channel is required for proper mucin secretion and link cystic fibrosis with its mucus phenotype. J. Exp. Med. 209, 1263-1272 (2012).

19. Johansson, M.E.V. et al. Bacteria penetrate the normally impenetrable inner colon mucus layer in both murine colitis models and in patients with ulcerative colitis. Gut 213, 281-291 (2014)

20. Jakobsson, H.E. et al. The gut microbiota composition impairs the colon inner mucus layer barrier. EMBO Rep online (2014).

21. Gum, J.R., Hicks, J.W., Toribara, N.W., Siddiki, B. \& Kim, Y.S. Molecular cloning of human intestinal mucin (MUC2) cDNA. Identification of the amino terminus and overall sequence similarity to prepro-von Willebrand factor. J. Biol. Chem. 269, 2440-2446 (1994).

22. Ambort, D. et al. Calcium and $\mathrm{pH}$-dependent packing and release of the gel-forming MUC2 mucin. Proc. Natl. Acad. Sci. USA 109, 5645-5650 (2012).

23. Barker, N. Adult intestinal stem cells: critical drivers of epithelial homeostasis and regeneration. Nat. Rev. Mol. Cell Biol. 15, 19-33 (2014).

24. Clevers, $\mathrm{H}$. The intestinal crypt, a prototype stem cell compartment. Cell 154, 274-284 (2013).

25. Gregorieff, A. et al. The Ets-domain transcription factor Spdef promotes maturation of goblet and paneth cells in the intestinal epithelium. Gastroenterology 137, 1333-1345 (2009).

26. Yu, K., Lujan, R., Marmorstein, A., Gabriel, S. \& Hartzell, H.C. Bestrophin-2 mediates bicarbonate transport by goblet cells in mouse colon. J. Clin. Invest. 120, 1722-1735 (2010).

27. Johansson, M.E.V. Fast renewal of the distal colonic mucus layers by the surface goblet cells as measured by in vivo labeling of mucin glycoproteins. PLoS One 7, e41009 (2012).

28. Specian, R.D. \& Neutra, M.R. Regulation of intestinal goblet cell secretion. I. Role of parasympathetic stimulation. Am. J. Physiol. 242, G370-G379 (1982).

29. Nilsson, H.E. et al. Intestinal MUC2 mucin supramolecular topology by packing and release resting on D3 domain assembly. J. Mol. Biol. 426, 2567-2579 (2014).
30. Ridley, C. et al. Assembly of the respiratory mucin MUC5B: a new model for a gel-forming mucin. J. Biol. Chem. 289, 16409-16420 (2014).

31. Verdugo, P. Mucin exocytosis. Am. Rev. Resp. Dis. 144, S33-S37 (1991).

32. Adler, K.B., Tuvim, M.J. \& Dickey, B.F. Regulated mucin secretion from airway epithelial cells. Front. Endocrinol. 4, 129 (2013).

33. Specian, D. \& Neutra, M.R. Mechanism of rapid mucus secretion in goblet cells stimulated by acetylcholine. J. Cell Biol. 85, 626-640 (1980).

34. Barbour, W.M. \& Hopwood, D. Uptake of cationized ferritin by colonic epithelium. J. Pathol. 139, 167-178 (1983)

35. Colony, P.C. \& Specian, R.D. Endocytosis and vesicular traffic in fetal and adult colonic goblet cells. Anat. Rec. 218, 365-372 (1987).

36. Herzog, V. Pathways of endocytosis in secretory cells. Trends Biochem. Sci. 6, 319-322 (1981).

37. Patel, K.K. et al. Autophagy proteins control goblet cell function by potentiating reactive oxygen species production. EMBO J. 32, 3130-3144 (2013).

38. Levine, B., Mizushima, N. \& Virgin, H.W. Autophagy in immunity and inflammation. Nature 469, 323-335 (2011).

39. Lamkanfi, M. \& Dixit, V.M. Mechanisms and functions of inflammasomes. Cell 157, 1013-1022 (2014).

40. Martinon, F., Burns, K. \& Tschopp, J. The inflammasome: a molecular platform triggering activation of inflammatory caspases and processing of proll-beta. Mol. Cell 10, 417-426 (2002).

41. Chen, G.Y., Liu, M., Wang, F., Bertin, J. \& Nunez, G. A functional role for Nlrp6 in intestinal inflammation and tumorigenesis. J. Immunol. 186, 7187-7194 (2011).

42. Elinav, E. et al. NLRP6 inflammasome regulates colonic microbial ecology and risk for colitis. Cell 145, 745-757 (2011).

43. Sellin, M. et al. Epithelium-intrinsic NAIP/NLRC4 inflammasome drives infected enterocyte expulsion to restrict salmonella replication in the intestinal mucosa. Cell Host Microbe 16, 237-248 (2014).

44. Wlodarska, M. et al. NLRP6 inflammasome orchestrates the colonic hostmicrobial interpace by regulating goblet cell mucus secretion. Cell 156, 1045-1059 (2014).

45. Neutra, M.R., O'Malley, L.J. \& Specian, R.D. Regulation of intestinal goblet cell secretion. II. A survey of potentid secretugogues. Am. J. Physiol. Gastroint. Liver Physiol. 242, G380-G387 (1982).

46. Halm, D.R. \& Halm, S.T. Secretagogue response of goblet cells and columnar cells in human colonic crypts. Am. J. Physiol. Cell Physiol. 278, C212-C233 (2000).

47. Gustafsson, J.K., Ermund, A., Johansson, M.E.V., Schutte, A., Hansson, G.C. \& Sjovall, H. An ex vivo method for studying mucus formation, properties and thickness in human colonic biopsies and mouse small and large intestinal explants. Am. J. Physiol. Gastrointest. Liver. Physiol. 302, G430-G438 (2012)

48. Halm, D.R. \& Halm, S.T. Secretagogue response of goblet cells and columnar cells in human colonic crypts. Am. J. Physiol. Cell Physiol. 278, C212-C233 (2000).

49. Garcia, M.A., Yang, N. \& Quinton, P.M. Normal mouse intestinal mucus release requires cystic fibrosis transmembrane regulator-dependent bicarbonate secretion. J. Clin. Invest. 119, 2613-2622 (2009).

50. Grootjans, J. et al. Ischemia-induced mucus barrier loss and bacterial penetration are rapidly counteracted by increased goblet cell secretory activity in human and rat colon. Gut 62, 250-258 (2013).

51. McDole, J.R. et al. Goblet cells deliver luminal antigen to CD103+ dendritic cells in the small intestine. Nature 483, 345-349 (2012).

52. Howe, S.E., Lickteig, D.J., Plunkett, K.N., Ryerse, J.S. \& Konjufca, V. The uptake of soluble and particulate antigens by epithelial cells in the mouse small intestine. PLoS One 9, e86656 (2014).

53. Knoop, K.A., McDonald, K.G., McCrate, S., McDole, J.R. \& Newberry, R.D. Microbial sensing by goblet cells controls immune surveillance of luminal antigens in the colon Mucosal Immunol. 8, 198-210 (2015).

54. Marillier, R.G. et al. IL-4/IL-13 independent goblet cell hyperplasia in experimental helminth infections. BMC Immunol. 9, 11 (2008).

55. Oeser, K., Schwartz, C. \& Voehringer, D. Conditional IL-4/IL-13-deficient mice reveal a critical role of innate immune cells for protective immunity against gastrointestinal helminths. Mucosal Immunol. doi:10.1038/mi.2014.101 (2014).

56. Finkelman, F.D. et al. Interleukin-4- and interleukin-13-mediated host protection against intestinal nematode parasites. Immunol. Rev. 201, 139-155 (2004). 
57. Steenwinckel, V.R. et al. IL-9 promotes IL-13-dependent paneth cell hyperplasia and up-regulation of innate immunity mediators in intestinal mucosa. J. Immunol. 182, 4737-4743 (2009).

58. Whitsett, J.A. \& Alenghat, T. Respiratory epithelial cells orchestrate pulmonary innate immunity. Nature Immunol. 16, 27-35 (2015).

59. Chen, G. et al. SPDEF is required for mouse pulmonary goblet cell differentiation and regulates a network of genes associated with mucus production. J Clin Invest 119, 2914-2924 (2009).

60. Johansson, M.E.V. \& Hansson, G.C. Is the intestinal goblet cell a major immune cell?. Cell Host Microbe 15, 251-252 (2014).

61. Turner, J.E., Stockinger, B. \& Helmby, H. IL-22 mediates goblet cell hyperplasia and worm expulsion in intestinal helminth infection. PLOS Pathog. 9, e1003698 (2013).

62. Martino, M.B. et al. The ER stress transducer IRE1beta is required for airway epithelial mucin production. Mucosal Immunol. 6, 639-654 (2012).

63. Tsuru, A. et al. Negative feedback by IRE1beta optimizes mucin production in goblet cells. Proc. Natl. Acad. Sci. USA 110, 2864-2869 (2013).

64. Hetz, C. The unfolded protein response: controlling cell fate decisions under ER stress and beyond. Nat. Rev. Mol. Cell Biol. 13, 89-102 (2012).

65. Heazlewood, C.K. et al. Aberrant mucin assembly in mice causes endoplasmic reticulum stress and spontaneous inflammation resembling ulcerative colitis. PLoS Med. 5, e54 (2008).

66. Eri, R.D. et al. An intestinal epithelial defect conferring ER stress results in inflammation involving both innate and adaptive immunity. Mucosal Immunol. 4, 354-364 (2011).

67. Colonna, M. Interleukin-22-producing natural killer cells and lymphoid tissue inducer-like cells in mucosal immunity. Immunity 31, 15-23 (2009).

68. Wang, X. et al. Interleukin-22 alleviates metabolic disorders and restores mucosal immunity in diabetes. Nature 514, 237-241 (2014).
69. Hasnain, S.Z. et al. Glycemic control in diabetes is restored by therapeutic manipulation of cytokines that regulate beta cell stress. Nat. Med. 20, 1417-1426 (2014).

70. Kuhn, R., Löhler, J., Rennick, D., Rajewsky, K. \& Muller, W. Interleukin-10deficient mice develop chronic enterocolitis. Cell 75, 263-274 (1993).

71. Spencer, S.D. et al. The orphan receptor CRF2-4 is an essential subunit of the interleukin 10 receptor. J. Exp. Med. 187, 571-578 (1998).

72. Sellon, R.K. et al. Resident enteric bacteria are necessary for development of spontaneous colitis and immune system activation in interleukin-10deficient mice. Infect. Immun. 66, 5224-5231 (1998).

73. Franke, A. et al. Replication of signals from recent studies of Crohn's disease identifies previously unknown disease loci for ulcerative colitis. Nat. Genet. 40, 713-715 (2008).

74. Franke, A. et al. Sequence variants in IL10, ARPC2 and multiple other loci contribute to ulcerative colitis susceptibility. Nat. Genet. 40, 1319-1323 (2008).

75. Begue, B. et al. Defective IL10 signaling defining a subgroup of patients with inflammatory bowel disease. Am. J. Gastroenterol. 106, 1544-1555 (2011).

76. Autschbach, F. et al. In situ expression of interleukin-10 in noninflamed human gut and in inflammatory bowel disease. Am. J. Pathol. 153 121-130 (1998).

77. Jarry, A. et al. Mucosal IL-10 and TGF-beta play crucial roles in preventing LPS-driven, IFN-gamma-mediated epithelial damage in human colon explants. J. Clin. Invest. 118, 1132-1142 (2008).

78. Steidler, L. et al. Treatment of murine colitis by lactococcus lactis secreting interleukin-10. Science 289, 1352-1355 (2000).

79. Hasnain, S.Z. et al. IL-10 promotes production of intestinal mucus by suppressing protein misfolding and endoplasmic reticulum stress in goblet cells. Gastroenterology 144, 357-368 (2013).

80. van der Sluis, M. et al. Combined defects in epithelial and immunoregulatory factors exacerbate the pathogenesis of inflammation: mucin 2interleukin 10-deficient mice. Lab. Invest. 88, 634-642 (2008). 